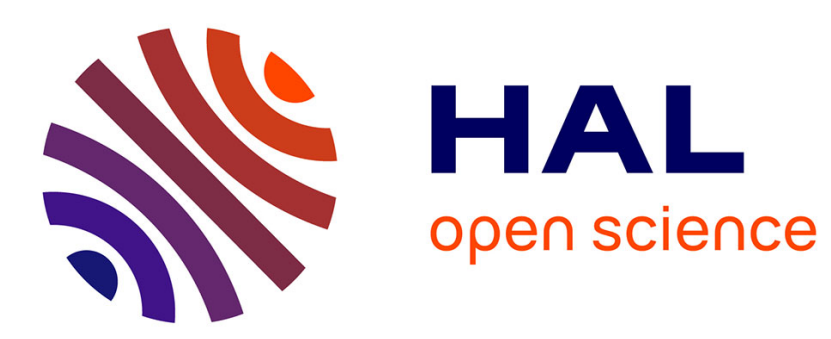

\title{
Estimation of solutions of observable nonlinear systems with disturbances
}

\author{
Frederic Mazenc, Emilia Fridman, Walid Djema
}

\section{To cite this version:}

Frederic Mazenc, Emilia Fridman, Walid Djema. Estimation of solutions of observable nonlinear systems with disturbances. Systems and Control Letters, 2015, 79 (May), pp.47-58. 10.1016/j.sysconle.2015.03.004 . hal-01257327

\section{HAL Id: hal-01257327 \\ https://inria.hal.science/hal-01257327}

Submitted on 18 Jan 2016

HAL is a multi-disciplinary open access archive for the deposit and dissemination of scientific research documents, whether they are published or not. The documents may come from teaching and research institutions in France or abroad, or from public or private research centers.
L'archive ouverte pluridisciplinaire HAL, est destinée au dépôt et à la diffusion de documents scientifiques de niveau recherche, publiés ou non, émanant des établissements d'enseignement et de recherche français ou étrangers, des laboratoires publics ou privés. 


\title{
Estimation of solutions of observable nonlinear systems with disturbances
}

\author{
Frédéric Mazenc, ${ }^{*} \quad$ Emilia Fridman, ${ }^{\dagger} \quad$ Walid Djema, ${ }^{\ddagger}$
}

March 6, 2015

\begin{abstract}
A family of continuous-time observable nonlinear systems with input and output is considered. A new technique of estimation of the state variables is proposed. It relies on the use of past values of the output, as done to construct some observers which converge in finite time, and on a recent technical result pertaining to the theory of the monotone systems. It applies to systems with additive disturbances and disturbances in the output. The nonlinear terms are not supposed to be globally Lipschitz, but it is requested that they depend only on the input and output variables.
\end{abstract}

Key Words: state estimation, delay, finite time observer, robustness

\section{Introduction}

The problem of estimating the value of the solutions of a system when some variables are not accessible by measurements is a fundamental problem, which has been addressed by many techniques in many contributions. Traditional state estimators, such as for instance the Luenberger observer [13], are very popular. They compute point estimates of the state from input-output data, possibly supplemented by an estimate of the dispersion of the possible values of the state around this point estimate. Guaranteed state estimators [2], [10, also known as set-membership estimators [1], [18, compute sets guaranteed to contain the actual value of the state if some hypotheses on the state perturbation and measurement noise are satisfied. Guaranteed state estimation can be traced back to the seminal work of F.C. Schweppe [23]. His idea was recursively to compute ellipsoids guaranteed to contain the actual state (of course, other types of containers than ellipsoids could and have been used). In the last two decades, a new technique of guaranteed state estimations has been proposed. It is based on tools called interval observers and is developed and applied in many studies, see, for instance, [8, 15], 21], 24], [16] and the references therein. Typically, interval observers bound the actual state between two functions which take advantage of the solutions of two deterministic and possibly coupled dynamical systems. A key feature of the interval observers is that they can be applied only when an approximate knowledge of the initial conditions (in terms of an upper and a lower bound) is known. By contrast with all the mentioned results, some observers make it possible to determine the value of the solutions of continuous-time systems in finite time. This is the case in particular of the observers designed in [4, [20, [11] [22] and [19] which converge to the solutions in finite time. Some papers present finite-time convergent observers for nonlinear systems that are linearizable up to output injection. This is case of [22] and [19]. All the above works consider systems without additive disturbances in the measurements. Recently finite time observers were designed for a class of nonlinear systems with unknown inputs [12. The latter results are confined to the case where the number of unknown inputs is not greater than the number of outputs.

*EPI INRIA DISCO, Laboratoire des Signaux et Systèmes (L2S, UMR CNRS 8506), CNRS, CentraleSupélec, Université Paris-Sud, 3 rue Joliot Curie, 91192, Gif-sur-Yvette, France. This research was partially supported by the iCODE institute, research project of the Idex Paris-Saclay. (e-mail: Frederic.MAZENC@lss.supelec.fr).

†School of Electrical Engineering, Tel Aviv University, 69974, Israel, (e-mail: emilia@eng.tau.ac.il). This work was partially supported by Israel Science Foundation (grants No $754 / 10$ and No 1128/14).

¥EPI INRIA DISCO, Laboratoire des Signaux et Systèmes (L2S, UMR CNRS 8506), CNRS, CentraleSupélec, Université Paris-Sud, 3 rue Joliot Curie, 91192, Gif-sur-Yvette, France. (e-mail: walid.djema@inria.fr). 
The aim of the present work is to propose a new approach of guaranteed state estimations for estimating state variables of nonlinear systems in the case where no approximate knowledge of the initial conditions is known and the systems have no monotonicity property. It is based on formulas incorporating past values of the input and the output of the studied system, which are reminiscent of the formulas proposed in [4, [12] and [22] and uses a recent technical result stated and proved in [17, which makes it possible to express a function without monotonicity property as another function (with domain of definition of dimension larger than the domain of the considered function) which is increasing with respect to some of its variables and decreasing with respect to others. We consider the systems for which two types of bounded deterministic time-varying disturbances are present: in the dynamics and in the output. Usually, these disturbances are present in applications; notice in particular that in general the measures are inaccurate. The results we obtain are of two types: some of the formulas of estimation we propose are functions of the past values of the input and the output only and have terms with distributed delays and others take advantage of dynamic extensions and have terms with pointwise constant delays only. In the absence of unknown uncertainties, the formulas we exhibit provide with the exact values of the solutions, after a finite time interval. When unknown disturbances are present and upper and lower bounded by known constant vectors, then, the formulas we propose give, after a finite time interval, upper and lower bounds for each component of the solutions, as interval observers do. The difference between these bounds is bounded as long as the disturbances are bounded. Finally, it is worth mentioning that we consider systems with nonlinear terms which are not supposed to be globally Lipschitz, but it is required that they depend only on the inputs and outputs.

The paper is organized as follows. The family of systems studied is presented in Section 2. The main results are stated and proved in Sections 3 and 4 . They are illustrated through examples in Section 5 . Concluding remarks are given in Section 6

\section{Notation, definitions and prerequisites.}

The notation will be simplified whenever no confusion can arise from the context. Any $k \times n$ matrix, whose entries are all 0 is simply denoted 0 . The Euclidean norm of vectors of any dimension and the induced norm of matrices of any dimension are denoted $|\cdot|$. All the inequalities must be understood componentwise (partial order of $\mathbb{R}^{r}$ ) i.e. $v_{a}=\left(v_{a 1}, \ldots, v_{a r}\right)^{\top} \in \mathbb{R}^{r}$ and $v_{b}=\left(v_{b 1}, \ldots, v_{b r}\right)^{\top} \in \mathbb{R}^{r}$ are such that $v_{a} \leq v_{b}$ if and only if, for all $i \in\{1, \ldots, r\}, v_{a i} \leq v_{b i}$. A symmetric matrix $M \in \mathbb{R}^{n \times n}$ is positive (resp. negative) semidefinite if for all vectors $v \in \mathbb{R}^{n}, v^{\top} M v \geq 0$ (resp. $\left.v^{\top} M v \leq 0\right)$. Then we denote $M \succeq 0$ (resp. $M \preceq 0$ ). A matrix $M \in \mathbb{R}^{n \times n}$ is said to be Schur stable if its spectral radius is smaller than 1. For two matrices $M=\left(m_{i j}\right) \in \mathbb{R}^{r \times s}$ and $N=\left(n_{i j}\right) \in \mathbb{R}^{r \times s}$ of same dimension, $\max \{M, N\}$ is the matrix where each entry is $\max \left\{m_{i j}, n_{i j}\right\}$. For a matrix $M \in \mathbb{R}^{r \times s}, M^{+}=\max \{M, 0\}, M^{-}=\max \{-M, 0\}$. A matrix $M \in \mathbb{R}^{r \times s}$ is said to be nonnegative if $M^{+}=M$. A sequence $\left(u_{i}\right)$ is nonnegative if for all integer $k, u_{k}$ is nonnegative. If a matrix $M$ is Metzler, then for all $t \geq 0, e^{M t} \geq 0$. For any continuous function $\varphi:[-\tau, \infty) \rightarrow \mathbb{R}^{n}$ and all $t \geq 0$, we define $\varphi_{t}$ by $\varphi_{t}(m)=\varphi(t+m)$ for all $m \in[-\tau, 0]$, i.e., $\varphi_{t} \in C_{\text {in }}$ is the translation operator.

\section{Family of studied systems}

Throughout the paper, we consider the nonlinear system

$$
\left\{\begin{array}{l}
\dot{x}(t)=F\left(x(t), u(t), \epsilon_{2}(t)\right) \\
y(t)=C x(t)+\epsilon_{1}(t)
\end{array}\right.
$$

where $x(t) \in \mathbb{R}^{n}$ is the state, and $C \in \mathbb{R}^{q \times n}, y(t) \in \mathbb{R}^{q}$ is the output, $u(t) \in \mathbb{R}^{p}$ is a possible known input, $F$ is a nonlinear function of class $C^{1}$, and $\epsilon_{1}:[0,+\infty) \rightarrow \mathbb{R}^{q}$ and $\epsilon_{2}:[0,+\infty) \rightarrow \mathbb{R}^{m}$ are disturbances, which are supposed to be piecewise continuous and bounded.

We introduce the following assumption:

Assumption A. The function $F$ is such that there exist a matrix $A \in \mathbb{R}^{n \times n}$ and a function $f$ of class $C^{1}$ such that, for all $x \in \mathbb{R}^{n}, u \in \mathbb{R}^{p}, \varepsilon \in \mathbb{R}^{m}$

$$
F(x, u, \varepsilon)=A x+f(C x, u, \varepsilon)
$$

and the pair $(A, C)$ is observable. 
We will also use the following assumption:

Assumption B. There are known constant vectors $\bar{\epsilon}_{1} \in \mathbb{R}^{q}, \underline{\epsilon}_{1} \in \mathbb{R}^{q}$ and $\bar{\epsilon}_{2} \in \mathbb{R}^{n}, \underline{\epsilon}_{2} \in \mathbb{R}^{n}$ such that, for $i=1,2$, and for all $t \geq 0$, the inequalities

$$
\underline{\epsilon}_{i} \leq \epsilon_{i}(t) \leq \bar{\epsilon}_{i}
$$

are satisfied.

\section{Discussion of the assumptions.}

- Notice that, along the trajectories of (1), $f\left(C x(t), u(t), \epsilon_{2}(t)\right)=f\left(y(t)-\epsilon_{1}(t), u(t), \epsilon_{2}(t)\right)$. Thus Assumption A implies that in the system (1), $f$ can be seen as a function which depends only on $y, u, \epsilon_{1}$ and $\epsilon_{2}$. Therefore, the family of systems (1) satisfying Assumption A belongs to the family of the systems affine in the unmeasured part of the state. For these systems, many constructions of asymptotic observers (see for instance [5]) and interval observers (see for instance [8], 7]) have been proposed.

- All the results of our paper can be extended straightforwardly to the case where the function $f$ depends on $t$ explicitly. For the sake of simplicity, we restrict ourselves to time-invariant systems.

- The decomposition (2) of the function $F$ is not unique. In particular, let us notice for later use that when Assumption A is satisfied, a matrix $A$ with real negative eigenvalues can always be selected.

- We prove in Appendix B that, under Assumption $\mathrm{A}$, for any selected matrix $A$, there is a matrix $L \in \mathbb{R}^{n \times q}$ such that the matrix

$$
H=A+L C \in \mathbb{R}^{n \times n}
$$

is Hurwitz and there is a constant $\tau>0$ such that the matrix

$$
e^{-\tau H}-e^{-\tau A} \in \mathbb{R}^{n \times n}
$$

is invertible. See also 4, where it is proved that $L$ can be chosen such that $e^{-\tau H}-e^{-\tau A}$ is invertible for arbitrarily small constants $\tau>0$.

- We will use the following notation:

$$
E_{\tau}=\left(e^{-\tau H}-e^{-\tau A}\right)^{-1} .
$$

- Assumption B is realistic and is frequently satisfied in practice. Moreover, it can be relaxed by allowing the bounds $\bar{\epsilon}_{i}, \underline{\epsilon}_{i}$ to depend on $t$. However, for the sake of simplicity, we restrict ourselves to the case where they are constant.

\section{Exact estimation}

The results of this section provide with exact estimations of the solutions in finite time, but they can be applied only when the functions $\epsilon_{1}$ and $\epsilon_{2}$ are known. Moreover, it is important to keep in mind that since Assumption A does not imply that the function $f$ is globally Lipschitz, the finite escape time-phenomenon may occur even if $u(t)$ is a bounded function. The results of the present section owe a great deal to the contributions 4$]$ and $[22$. However, they are very different from those of [4, which are devoted to linear systems without functions $\epsilon_{1}$ and $\epsilon_{2}$. The results in [22] are also concerned with systems without functions $\epsilon_{1}$ and $\epsilon_{2}$, but they apply to systems (1) when they satisfy Assumption A.

\subsection{Exact estimation, direct approach}

Let us state and prove the following result:

Theorem 1. Let the system (1) satisfy Assumption A. Let $L \in \mathbb{R}^{n \times q}$ and $\tau>0$ be such that the corresponding matrix $e^{-\tau H}-e^{-\tau A}$ is invertible. Then, for a given piecewise continuous input $u(t)$, any solution $x(t)$ of the system (1) which exists over $[0,+\infty)$ satisfies, for all $t \geq \tau$,

$$
\begin{aligned}
x(t)= & -E_{\tau} \int_{t-\tau}^{t} e^{(t-l-\tau) A} f\left(y(l)-\epsilon_{1}(l), u(l), \epsilon_{2}(l)\right) d l \\
& +E_{\tau} \int_{t-\tau}^{t} e^{(t-l-\tau) H}\left[f\left(y(l)-\epsilon_{1}(l), u(l), \epsilon_{2}(l)\right)-L y(l)\right] d l+E_{\tau} \int_{t-\tau}^{t} e^{(t-l-\tau) H} L \epsilon_{1}(l) d l .
\end{aligned}
$$


Proof. From the definition of the output $y$ and the definition of $H$, we deduce that the system (1) admits the following representations:

$$
\begin{aligned}
& \dot{x}(t)=A x(t)+f\left(y(t)-\epsilon_{1}(t), u(t), \epsilon_{2}(t)\right), \\
& \dot{x}(t)=H x(t)+f\left(y(t)-\epsilon_{1}(t), u(t), \epsilon_{2}(t)\right)-L y(t)+L \epsilon_{1}(t) .
\end{aligned}
$$

By integrating these two systems between two values $s_{1} \geq 0$ and $s_{2} \geq 0$, we obtain the equalities

$$
\begin{aligned}
& x\left(s_{1}\right)=e^{A\left(s_{1}-s_{2}\right)} x\left(s_{2}\right)+\int_{s_{2}}^{s_{1}} e^{A\left(s_{1}-l\right)} f\left(y(l)-\epsilon_{1}(l), u(l), \epsilon_{2}(l)\right) d l, \\
& x\left(s_{1}\right)=e^{H\left(s_{1}-s_{2}\right)} x\left(s_{2}\right)+\int_{s_{2}}^{s_{1}} e^{H\left(s_{1}-l\right)}\left[f\left(y(l)-\epsilon_{1}(l), u(l), \epsilon_{2}(l)\right)-L y(l)+L \epsilon_{1}(l)\right] d l .
\end{aligned}
$$

Now, consider a value $t \geq \tau$. Then selecting $s_{2}=t$ and $s_{1}=t-\tau$, the equalities $(9)$ give

$$
\begin{aligned}
& x(t-\tau)=e^{-\tau A} x(t)+\int_{t}^{t-\tau} e^{(t-l-\tau) A} f\left(y(l)-\epsilon_{1}(l), u(l), \epsilon_{2}(l)\right) d l, \\
& x(t-\tau)=e^{-\tau H} x(t)+\int_{t}^{t-\tau} e^{(t-l-\tau) H}\left[f\left(y(l)-\epsilon_{1}(l), u(l), \epsilon_{2}(l)\right)-L y(l)+L \epsilon_{1}(l)\right] d l .
\end{aligned}
$$

As an immediate consequence, we have

$$
\begin{aligned}
\left(e^{-\tau H}-e^{-\tau A}\right) x(t)= & \left.-\int_{t-\tau}^{t} e^{(t-l-\tau) A} f\left(y(l)-\epsilon_{1}(l), u(l)\right), \epsilon_{2}(l)\right) d l \\
& +\int_{t-\tau}^{t} e^{(t-l-\tau) H}\left[f\left(y(l)-\epsilon_{1}(l), u(l), \epsilon_{2}(l)\right)-L y(l)+L \epsilon_{1}(l)\right] d l .
\end{aligned}
$$

Since $e^{-\tau H}-e^{-\tau A}$ is invertible, we deduce that $(7)$ is satisfied.

\subsection{Exact estimation with dynamic extensions}

The formula (7) includes terms with distributed delays, which in practice can be difficult to evaluate. To overcome this drawback, we propose the result below, which relies on a formula without distributed terms. This theorem is reminiscent of the main results of [4] and 22.

Theorem 2. Let the system (1) satisfy Assumption A, let $L$ and $\tau$ be defined as in Theorem 1 and let $u$ be a piecewise continuous function. Consider the dynamic extensions

$$
\dot{\hat{x}}(t)=A \hat{x}(t)+f\left(y(t)-\epsilon_{1}(t), u(t), \epsilon_{2}(t)\right)
$$

and

$$
\dot{x}_{*}(t)=H x_{*}(t)+f\left(y(t)-\epsilon_{1}(t), u(t), \epsilon_{2}(t)\right)-L y(t)+L \epsilon_{1}(t) .
$$

where $y$ is the output of (1). Consider a solution $x(t)$ of (1) defined over $[0,+\infty)$. Then, for all $t \geq \tau$,

$$
x(t)=E_{\tau}\left(e^{-H \tau} x_{*}(t)-x_{*}(t-\tau)-e^{-A \tau} \hat{x}(t)+\hat{x}(t-\tau)\right) .
$$

Remark 1. When one of the eigenvalues of the matrix $A$ has a positive real part, then even when a bounded solution $x(t)$, a bounded input $u(t)$ and bounded functions $\epsilon_{i}(t)$ are considered, the system (12) admits exponentially unstable solutions. This can be a drawback in applied cases. Fortunately, as mentioned in the discussion of Assumptions A and $\mathrm{B}$, it is always possible to choose a decomposition of $F$ so that the corresponding matrix $A$ has real negative eigenvalues. Notice also that in the absence of disturbance, the system (13) is a classical observer for (1) when $A$ is Hurwitz.

Proof. Consider a solution $\left(\hat{x}(t), x_{*}(t)\right)$ of 12$\left.]-13\right)$ associated with the solution $x(t)$ defined over $[0,+\infty)$. Then, $f\left(y(t)-\epsilon_{1}(t), u(t), \epsilon_{2}(t)\right)$ and $f\left(y(t)-\epsilon_{1}(t), u(t), \epsilon_{2}(t)\right)-L y(t)+L \epsilon_{1}(t)$ are piecewise continuous functions of $t$ and are defined over $[0,+\infty)$. By integrating (12) and (13), we deduce that, for all constants $s_{1} \geq 0$ and $s_{2} \geq 0$, the equalities

$$
\begin{aligned}
\hat{x}\left(s_{1}\right) & =e^{A\left(s_{1}-s_{2}\right)} \hat{x}\left(s_{2}\right)+\int_{s_{2}}^{s_{1}} e^{A\left(s_{1}-l\right)} f\left(y(l)-\epsilon_{1}(l), u(l), \epsilon_{2}(l)\right) d l, \\
x_{*}\left(s_{1}\right) & =e^{H\left(s_{1}-s_{2}\right)} x_{*}\left(s_{2}\right)+\int_{s_{2}}^{s_{1}} e^{H\left(s_{1}-l\right)}\left[f\left(y(l)-\epsilon_{1}(l), u(l), \epsilon_{2}(l)\right)-L y(l)+L \epsilon_{1}(l)\right] d l
\end{aligned}
$$

are satisfied. It follows that, for all $t \geq \tau$,

$$
\begin{aligned}
\int_{t-\tau}^{t} e^{A(t-\tau-l)} f\left(y(l)-\epsilon_{1}(l), u(l), \epsilon_{2}(l)\right) d l & =e^{-A \tau} \hat{x}(t)-\hat{x}(t-\tau), \\
\int_{t-\tau}^{t} e^{H(t-\tau-l)}\left[f\left(y(l)-\epsilon_{1}(l), u(l), \epsilon_{2}(l)\right)-L y(l)+L \epsilon_{1}(l)\right] d l & =e^{-H \tau} x_{*}(t)-x_{*}(t-\tau) .
\end{aligned}
$$

From (7) and (16), it follows that, for all $t \geq \tau$,

$$
E_{\tau}^{-1} x(t)=e^{-H \tau} x_{*}(t)-x_{*}(t-\tau)-e^{-A \tau} \hat{x}(t)+\hat{x}(t-\tau) .
$$

This allows us to conclude. 


\section{Approximate estimation}

Theorems 1 and 2 give in finite time the exact value of any solution $x(t)$ of (1) that is defined for all $t \geq 0$. The limitation of these results is that they cannot be used when the disturbances $\epsilon_{1}$ and $\epsilon_{2}$ are unknown. The purpose of this section is to overcome this limitation by assuming only that the bounds $\bar{\epsilon}_{i}$ and $\underline{\epsilon}_{i}$ in Assumption B are known. To the best of our knowledge no result of the type of those proposed in this section are available in the literature.

Let us recall that Assumption A ensures that the matrix $A$ in the decomposition of $F$ can be chosen such that all its eigenvalues are negative real numbers, which implies that its Jordan form is Metzler and Hurwitz. Then there are an invertible matrix $R_{1} \in \mathbb{R}^{n \times n}$ and a Metzler and Hurwitz matrix $M_{1} \in \mathbb{R}^{n \times n}$ such that

$$
M_{1}=R_{1} A R_{1}^{-1} .
$$

For the same reason, there is a matrix $L \in \mathbb{R}^{n \times q}$ such that the matrix

$$
H=A+L C \in \mathbb{R}^{n \times n}
$$

has real negative eigenvalues and then there exist an invertible matrix $R_{2} \in \mathbb{R}^{n \times n}$ and a Metzler and Hurwitz matrix $M_{2} \in \mathbb{R}^{n \times n}$ such that

$$
M_{2}=R_{2} H R_{2}^{-1} .
$$

In this section, we consider the case where Assumption $\mathrm{A}$ is satisfied and the matrices $A$ and $L$ are selected as described above.

\subsection{Approximate estimation, direct approach}

Since the function $f$ is of class $C^{1}$, Lemma 2, proved in [17] and recalled in Appendix B ensures that there are Lipschitz continuous function $\varphi_{i}: \mathbb{R}^{q} \times \mathbb{R}^{q} \times \mathbb{R}^{p} \times \mathbb{R}^{m} \times \mathbb{R}^{m} \rightarrow \mathbb{R}^{n}, i=1,2$, such that

$$
\varphi_{1}\left(y, \epsilon_{2}, y, \epsilon_{2}, u\right)=-R_{1} f\left(y, u, \epsilon_{2}\right) \quad, \quad \varphi_{2}\left(y, \epsilon_{2}, y, \epsilon_{2}, u\right)=R_{2} f\left(y, u, \epsilon_{2}\right) \quad, \quad \forall\left(y, u, \epsilon_{2}\right) \in \mathbb{R}^{q \times p \times m}
$$

and $\varphi_{1}\left(a_{1}, a_{2}, b_{1}, b_{2}, u\right)$ and $\varphi_{2}\left(a_{1}, a_{2}, b_{1}, b_{2}, u\right)$ are increasing with respect to $a_{1}$ and $a_{2}$ and decreasing with respect to $b_{1}$ and $b_{2}$. Next, we define the following functions for $i=1,2$,

$$
\begin{gathered}
\bar{\psi}_{i}(y, u)=\varphi_{i}\left(y-\underline{\epsilon}_{1}, \bar{\epsilon}_{2}, y-\bar{\epsilon}_{1}, \underline{\epsilon}_{2}, u\right), \underline{\psi}_{i}(y, u)=\varphi_{i}\left(y-\bar{\epsilon}_{1}, \underline{\epsilon}_{2}, y-\underline{\epsilon}_{1}, \bar{\epsilon}_{2}, u\right), \\
\epsilon_{3}(t)=e^{-\tau M_{2}} R_{2} L \epsilon_{1}(t),
\end{gathered}
$$

the matrices

$$
F_{\tau}=E_{\tau} R_{1}^{-1} e^{-\tau M_{1}}, G_{\tau}=E_{\tau} R_{2}^{-1} e^{-\tau M_{2}}
$$

and the operators

$$
\begin{gathered}
\overline{\mathcal{T}}_{1}\left(y_{t}, u_{t}\right)=F_{\tau}^{+} \int_{t-\tau}^{t} e^{(t-l) M_{1}} \bar{\psi}_{1}(y(l), u(l)) d l-F_{\tau}^{-} \int_{t-\tau}^{t} e^{(t-l) M_{1}} \psi_{1}(y(l), u(l)) d l, \\
\mathcal{T}_{1}\left(y_{t}, u_{t}\right)=F_{\tau}^{+} \int_{t-\tau}^{t} e^{(t-l) M_{1}} \psi_{1}(y(l), u(l)) d l-F_{\tau}^{-} \int_{t-\tau}^{t} e^{(t-l) M_{1}} \overline{\bar{\psi}}_{1}(y(l), u(l)) d l, \\
\overline{\mathcal{T}}_{2}\left(y_{t}, u_{t}\right)=G_{\tau}^{+} \int_{t-\tau}^{t} e^{(t-l) M_{2}} \overline{\bar{\psi}}_{2}(y(l), u(l)) d l-G_{\tau}^{-} \int_{t-\tau}^{t} e^{(t-l) M_{2}} \underline{\psi}_{2}(y(l), u(l)) d l, \\
\mathcal{T}_{2}\left(y_{t}, u_{t}\right)=G_{\tau}^{+} \int_{t-\tau}^{t} e^{(t-l) M_{2}} \underline{\psi}_{2}(y(l), u(l)) d l-G_{\tau}^{-} \int_{t-\tau}^{t} e^{(t-l) M_{2}} \overline{\bar{\psi}}_{2}(y(l), u(l)) d l, \\
\overline{\mathcal{S}}\left(y_{t}, u_{t}\right)=\overline{\mathcal{T}}_{1}\left(y_{t}, u_{t}\right)+\overline{\mathcal{T}}_{2}\left(y_{t}, u_{t}\right), \\
\underline{\mathcal{S}}\left(y_{t}, u_{t}\right)=\underline{\mathcal{T}}_{1}\left(y_{t}, u_{t}\right)+\underline{\mathcal{T}}_{2}\left(y_{t}, u_{t}\right) .
\end{gathered}
$$

We are ready to state and prove the following result:

Theorem 3. Let the system (1) satisfy Assumptions $A$ and $B$ and let the matrix $A$ be such that, for some matrices $L$, $M_{1}, M_{2}, R_{1}, R_{2}$ and a constant $\tau>0$ the equalities (18) and (20) are satisfied and $E_{\tau}$ defined in (6) is well-defined. Let $u$ be piecewise continuous and consider a solution (1) defined over $[0,+\infty)$. Then, for all $t \geq \tau$, the inequalities

$$
\underline{\mathcal{S}}\left(y_{t}, u_{t}\right)-E_{\tau} \int_{t-\tau}^{t} e^{(t-l-\tau) H} L y(l) d l+\epsilon_{S} \leq x(t) \leq \overline{\mathcal{S}}\left(y_{t}, u_{t}\right)-E_{\tau} \int_{t-\tau}^{t} e^{(t-l-\tau) H} L y(l) d l+\epsilon_{L}
$$


where

$$
\begin{aligned}
\epsilon_{L}= & {\left[\left(E_{\tau} R_{2}^{-1}\right)^{+} M_{3}\left(e^{-\tau M_{2}} R_{2} L\right)^{+}+\left(E_{\tau} R_{2}^{-1}\right)^{-} M_{3}\left(e^{-\tau M_{2}} R_{2} L\right)^{-}\right] \bar{\epsilon}_{1} } \\
& -\left[\left(E_{\tau} R_{2}^{-1}\right)^{+} M_{3}\left(e^{-\tau M_{2}} R_{2} L\right)^{-}+\left(E_{\tau} R_{2}^{-1}\right)^{-} M_{3}\left(e^{-\tau M_{2}} R_{2} L\right)^{+}\right] \epsilon_{1}, \\
\epsilon_{S}= & {\left[\left(E_{\tau} R_{2}^{-1}\right)^{+} M_{3}\left(e^{-\tau M_{2}} R_{2} L\right)^{+}+\left(E_{\tau} R_{2}^{-1}\right)^{-} M_{3}\left(e^{-\tau M_{2}} R_{2} L\right)^{-}\right] \epsilon_{1} } \\
& -\left[\left(E_{\tau} R_{2}^{-1}\right)^{+} M_{3}\left(e^{-\tau M_{2}} R_{2} L\right)^{-}+\left(E_{\tau} R_{2}^{-1}\right)^{-} M_{3}\left(e^{-\tau M_{2}} R_{2} L\right)^{+}\right] \bar{\epsilon}_{1},
\end{aligned}
$$

with $M_{3}=M_{2}^{-1}\left(e^{\tau M_{2}}-I\right)$, are satisfied.

Proof. From (7) and the definitions of $\varphi_{1}$ and $\varphi_{2}$ in (21), we deduce immediately that, for all $t \geq \tau$,

$$
\begin{aligned}
x(t)= & E_{\tau} \int_{t-\tau}^{t} e^{(t-l-\tau) A} R_{1}^{-1} \varphi_{1}\left(y(l)-\epsilon_{1}(l), \epsilon_{2}(l), y(l)-\epsilon_{1}(l), \epsilon_{2}(l), u(l)\right) d l \\
& +E_{\tau} \int_{t-\tau}^{t} e^{(t-l-\tau) H}\left[R_{2}^{-1} \varphi_{2}\left(y(l)-\epsilon_{1}(l), \epsilon_{2}(l), y(l)-\epsilon_{1}(l), \epsilon_{2}(l), u(l)\right)-L y(l)\right] d l \\
& +E_{\tau} \int_{t-\tau}^{t} e^{(t-l-\tau) H} L \epsilon_{1}(l) d l .
\end{aligned}
$$

From 18 and 201 , it follows that

$$
\begin{aligned}
x(t)= & \left.F_{\tau} \int_{t-\tau}^{t} e^{(t-l) M_{1}} \varphi_{1}\left(y(l)-\epsilon_{1}(l), \epsilon_{2}(l), y(l)-\epsilon_{1}(l), \epsilon_{2}(l), u(l)\right)\right) d l \\
& \left.+G_{\tau} \int_{t-\tau}^{t} e^{(t-l) M_{2}} \varphi_{2}\left(y(l)-\epsilon_{1}(l), \epsilon_{2}(l), y(l)-\epsilon_{1}(l), \epsilon_{2}(l), u(l)\right)\right) d l-E_{\tau} \int_{t-\tau}^{t} e^{(t-l-\tau) H} L y(l) d l \\
& +E_{\tau} R_{2}^{-1} \int_{t-\tau}^{t} e^{(t-l) M_{2}} \epsilon_{3}(l) d l .
\end{aligned}
$$

From Assumption B and the monotonicity properties of the functions $\varphi_{1}$ and $\varphi_{2}$, it follows that, for $i=1,2$, the inequalities

$$
\left.\underline{\psi}_{i}(y(t), u(t)) \leq \varphi_{i}\left(y(t)-\epsilon_{1}(t), \epsilon_{2}(t), y(t)-\epsilon_{1}(t), \epsilon_{2}(t), u(t)\right)\right) \leq \bar{\psi}_{i}(y(t), u(t))
$$

hold for all $t \geq 0$. Using the fact that the matrices $M_{1}$ and $M_{2}$ are Metzler and the definitions of $\underline{\psi}_{i}$ and $\bar{\psi}_{i}$, it follows that, for $i=1,2$, and all $t \geq \tau$, the inequalities

$$
\int_{t-\tau}^{t} e^{(t-l) M_{i}} \underline{\psi}_{i}(y(l), u(l)) d l \leq \int_{t-\tau}^{t} e^{(t-l) M_{i}} \varphi_{i}(\cdot) d l \leq \int_{t-\tau}^{t} e^{(t-l) M_{i}} \bar{\psi}_{i}(y(l), u(l)) d l
$$

with $\varphi_{i}(\cdot)=\varphi_{i}\left(y(l)-\epsilon_{1}(l), \epsilon_{2}(l), y(l)-\epsilon_{1}(l), \epsilon_{2}(l), u(l)\right)$ are satisfied. We deduce easily that for all $t \geq \tau$,

$$
\begin{aligned}
& \underline{\mathcal{T}}_{1}\left(y_{t}, u_{t}\right) \leq F_{\tau} \int_{t-\tau}^{t} e^{(t-l) M_{1}} \varphi_{1}(\cdot) d l \leq \overline{\mathcal{T}}_{1}\left(y_{t}, u_{t}\right), \\
& \underline{\mathcal{T}}_{2}\left(y_{t}, u_{t}\right) \leq G_{\tau} \int_{t-\tau}^{t} e^{(t-l) M_{2}} \varphi_{2}(\cdot) d l \leq \overline{\mathcal{T}}_{2}\left(y_{t}, u_{t}\right) .
\end{aligned}
$$

In Appendix $\mathrm{A}$ we prove that, for all $t \geq \tau$,

$$
\epsilon_{S} \leq E_{\tau} R_{2}^{-1} \int_{t-\tau}^{t} e^{(t-l) M_{2}} \epsilon_{3}(l) d l \leq \epsilon_{L} .
$$

It follows from $(29),(32)$ and $(33)$ that $(26)$ is satisfied.

\subsection{Approximate estimation with dynamic extensions}

The motivations of Theorem 2 also motivate the following result:

Theorem 4. Let the system (1) satisfy the conditions in Theorem 3. Let u(t) be piecewise continuous and consider a solution (1) defined over $[0,+\infty)$. Let us introduce several dynamic extensions:

$$
\begin{aligned}
& \dot{z}_{a}(t)=H z_{a}(t)+L y(t), \\
& \dot{z}_{b}(t)=M_{1} z_{b}(t)+\bar{\psi}_{1}(y(t), u(t)), \\
& \dot{z}_{c}(t)=M_{1} z_{c}(t)+\underline{\psi}_{1}(y(t), u(t)), \\
& \dot{z}_{d}(t)=M_{2} z_{d}(t)+\overline{\bar{\psi}}_{2}(y(t), u(t)), \\
& \dot{z}_{e}(t)=M_{2} z_{e}(t)+\underline{\psi}_{2}(y(t), u(t)),
\end{aligned}
$$

where $y$ and $u$ are the output and input in (1). Then, for all $t \geq \tau$, the inequalities

$$
\underline{\mathcal{V}}\left(Z_{t}\right) \leq x(t) \leq \overline{\mathcal{V}}\left(Z_{t}\right)
$$


with $Z=\left(z_{a}, z_{b}, z_{c}, z_{d}, z_{e}\right)$ and

$$
\begin{aligned}
\overline{\mathcal{V}}\left(Z_{t}\right)= & F_{\tau}^{+}\left[z_{b}(t)-e^{\tau M_{1}} z_{b}(t-\tau)\right]+F_{\tau}^{-}\left[e^{\tau M_{1}} z_{c}(t-\tau)-z_{c}(t)\right]+G_{\tau}^{+}\left[z_{d}(t)-e^{\tau M_{2}} z_{d}(t-\tau)\right] \\
& +G_{\tau}^{-}\left[e^{\tau M_{2}} z_{e}(t-\tau)-z_{e}(t)\right]+E_{\tau} e^{-\tau H}\left[e^{\tau H} z_{a}(t-\tau)-z_{a}(t)\right]+\epsilon_{L},
\end{aligned}
$$

where $\epsilon_{S}$ and $\epsilon_{L}$ are the constants given in 27), are satisfied.

Proof. Consider a solution $x(t)$ of (1) defined over $[0,+\infty)$. Since $y$ and $u$ are bounded on any compact time interval, by integrating (34), we obtain, for all $t \geq \tau$,

$$
\begin{aligned}
& z_{a}(t)=e^{\tau H} z_{a}(t-\tau)+\int_{t-\tau}^{t} e^{(t-l) H} L y(l) d l, \\
& z_{b}(t)=e^{\tau M_{1}} z_{b}(t-\tau)+\int_{t-\tau}^{t} e^{(t-l) M_{1}} \bar{\psi}_{1}(y(l), u(l)) d l, \\
& z_{c}(t)=e^{\tau M_{1}} z_{c}(t-\tau)+\int_{t-\tau}^{t} e^{(t-l) M_{1}} \psi_{1}(y(l), u(l)) d l, \\
& z_{d}(t)=e^{\tau M_{2}} z_{d}(t-\tau)+\int_{t-\tau}^{t} e^{(t-l) M_{2}} \overline{\bar{\psi}}_{2}(y(l), u(l)) d l, \\
& z_{e}(t)=e^{\tau M_{2}} z_{e}(t-\tau)+\int_{t-\tau}^{t} e^{(t-l) M_{2}} \underline{\psi}_{2}(y(l), u(l)) d l .
\end{aligned}
$$

These equalities rewrite as

$$
\begin{aligned}
\int_{t-\tau}^{t} e^{(t-l) H} L y(l) d l & =z_{a}(t)-e^{\tau H} z_{a}(t-\tau), \\
\int_{t-\tau}^{t} e^{(t-l) M_{1}} \bar{\psi}_{1}(y(l), u(l)) d l & =z_{b}(t)-e^{\tau M_{1}} z_{b}(t-\tau), \\
\int_{t-\tau}^{t} e^{(t-l) M_{1}} \underline{\psi}_{1}(y(l), u(l)) d l & =z_{c}(t)-e^{\tau M_{1}} z_{c}(t-\tau), \\
\int_{t-\tau}^{t} e^{(t-l) M_{2}} \bar{\psi}_{2}(y(l), u(l)) d l & =z_{d}(t)-e^{\tau M_{2}} z_{d}(t-\tau), \\
\int_{t-\tau}^{t} e^{(t-l) M_{2}} \underline{\psi}_{2}(y(l), u(l)) d l & =z_{e}(t)-e^{\tau M_{2}} z_{e}(t-\tau) .
\end{aligned}
$$

It follows that, for all $t \geq \tau$, the equalities

$$
\begin{gathered}
\overline{\mathcal{T}}_{1}\left(y_{t}, u_{t}\right)=F_{\tau}^{+}\left[z_{b}(t)-e^{\tau M_{1}} z_{b}(t-\tau)\right]+F_{\tau}^{-}\left[e^{\tau M_{1}} z_{c}(t-\tau)-z_{c}(t)\right], \\
\mathcal{T}_{1}\left(y_{t}, u_{t}\right)=F_{\tau}^{+}\left[z_{c}(t)-e^{\tau M_{1}} z_{c}(t-\tau)\right]+F_{\tau}^{-}\left[e^{\tau M_{1}} z_{b}(t-\tau)-z_{b}(t)\right], \\
\overline{\overline{\mathcal{T}}}_{2}\left(y_{t}, u_{t}\right)=G_{\tau}^{+}\left[z_{d}(t)-e^{\tau M_{2}} z_{d}(t-\tau)\right]+G_{\tau}^{-}\left[e^{\tau M_{2}} z_{e}(t-\tau)-z_{e}(t)\right], \\
\mathcal{\mathcal { T }}_{2}\left(y_{t}, u_{t}\right)=G_{\tau}^{+}\left[z_{e}(t)-e^{\tau M_{2}} z_{e}(t-\tau)\right]+G_{\tau}^{-}\left[e^{\tau M_{2}} z_{d}(t-\tau)-z_{d}(t)\right] \\
-E_{\tau} \int_{t-\tau}^{t} e^{(t-l-\tau) H} L y(l) d l=-E_{\tau} e^{-\tau H}\left[z_{a}(t)-e^{\tau H} z_{a}(t-\tau)\right] .
\end{gathered}
$$

are satisfied. Evidently, Theorem 3 applies. Then from the inequalities $(26)$ and the equalities $(40)$, we deduce that the inequalities (35) are satisfied. 


\section{Illustration}

\subsection{First example}

In this section, we illustrate Theorems 2 and 4 with the two dimensional system:

$$
\left\{\begin{aligned}
\dot{x}_{1} & =x_{2}+x_{1}^{2} \sin \left(x_{1}\right)+\epsilon_{2}, \\
\dot{x}_{2} & =x_{1}^{2} \sin \left(x_{1}\right)+\epsilon_{2}, \\
y & =x_{1}+\epsilon_{1} .
\end{aligned}\right.
$$

This system is of the form 11 with $C=\left(\begin{array}{ll}1 & 0\end{array}\right)$. Notice that the function $x_{1}^{2} \sin \left(x_{1}\right)$ is not globally Lipschitz. We can choose

$$
A=\left[\begin{array}{ll}
-\frac{5}{2} & 1 \\
-\frac{3}{2} & 0
\end{array}\right], \quad f\left(y, u, \epsilon_{2}\right)=\left(\begin{array}{c}
y^{2} \sin (y)+\frac{5}{2} y+\epsilon_{2} \\
y^{2} \sin (y)+\frac{3}{2} y+\epsilon_{2}
\end{array}\right) .
$$

Notice that $A$ is Hurwitz, the pair $(A, C)$ is observable and the function $f$ is of class $C^{1}$. Next, let us check that Assumption A3 is satisfied. Let

$$
R_{1}=\left[\begin{array}{rc}
3 & -2 \\
-2 & 2
\end{array}\right]
$$

Then

$$
R_{1}^{-1}=\left[\begin{array}{ll}
1 & 1 \\
1 & \frac{3}{2}
\end{array}\right]
$$

and

$$
R_{1} A R_{1}^{-1}=M_{1}
$$

with

$$
M_{1}=\left[\begin{array}{cc}
-\frac{3}{2} & 0 \\
0 & -1
\end{array}\right] \text {. }
$$

Selecting $L=-\left(\begin{array}{c}\frac{5}{2} \\ \frac{9}{2}\end{array}\right)$, we obtain

$$
H=\left[\begin{array}{ll}
-5 & 1 \\
-6 & 0
\end{array}\right]
$$

Notice that

$$
R_{2}=\left[\begin{array}{rc}
-2 & 1 \\
3 & -1
\end{array}\right]
$$

is such that $R_{2}^{-1}=\left[\begin{array}{ll}1 & 1 \\ 3 & 2\end{array}\right]$ and

$$
R_{2} H R_{2}^{-1}=M_{2}
$$

with

$$
M_{2}=\left[\begin{array}{cc}
-2 & 0 \\
0 & -3
\end{array}\right] .
$$

Therefore Assumption A3 is satisfied. Next, let us check that Assumption A2 is satisfied.

$$
\begin{aligned}
e^{-\tau H}-e^{-\tau A} & =R_{2}^{-1} e^{-\tau M_{2}} R_{2}-R_{1}^{-1} e^{-\tau M_{1}} R_{1} \\
& =\left[\begin{array}{cc}
3 e^{3 \tau}-2 e^{2 \tau}-3 e^{\frac{3 \tau}{2}}+2 e^{\tau} & e^{2 \tau}-e^{3 \tau}+2 e^{\frac{3 \tau}{2}}-2 e^{\tau} \\
6 e^{3 \tau}-6 e^{2 \tau}-3 e^{\frac{3 \tau}{2}}+3 e^{\tau} & 3 e^{2 \tau}-2 e^{3 \tau}+2 e^{\frac{3 \tau}{2}}-3 e^{\tau}
\end{array}\right] .
\end{aligned}
$$

This matrix is invertible when $\tau$ is sufficiently large. Therefore Assumption A2 is satisfied. Defining

$$
\delta_{\tau}=\operatorname{det}\left(e^{-\tau H}-e^{-\tau A}\right),
$$

we have

$$
\delta_{\tau}=2 e^{4 \tau}-3 e^{3 \tau}+e^{5 \tau}+e^{\frac{5 \tau}{2}}+2 e^{\frac{7 \tau}{2}}-3 e^{\frac{9 \tau}{2}} .
$$


Then,

$$
E_{\tau}=\left(e^{-\tau H}-e^{-\tau A}\right)^{-1}=\left[\begin{array}{cc}
\frac{4 e^{\frac{\tau}{2}}+2 e^{\tau}+3}{e^{2 \tau}-e^{3 \tau}-e^{\frac{3 \tau}{2}}+e^{\frac{5 \tau}{2}}} & -\frac{2 e^{\frac{\tau}{2}}+e^{\tau}+2}{e^{2 \tau}-e^{3 \tau}-e^{\frac{3 \tau}{2}}+e^{\frac{5 \tau}{2}}} \\
\frac{-3\left(2 e^{\frac{3 \tau}{2}}+2 e^{\tau}-1\right)}{2 e^{2 \tau}-2 e^{3 \tau}-e^{\frac{3 \tau}{2}}+e^{\frac{7 \tau}{2}}} & \frac{e^{\frac{\tau}{2}}+3 e^{\frac{3 \tau}{2}}+3 e^{\tau}-2}{2 e^{2 \tau}-2 e^{3 \tau}-e^{\frac{3 \tau}{2}}+e^{\frac{7 \tau}{2}}}
\end{array}\right] .
$$

We apply Theorem 2 with $\epsilon_{1}(t)=\frac{1}{9} \sin (t), \epsilon_{2}(t)=\frac{1}{2} \sin \left(t^{2}\right)$. We obtain the output $y(t)$ shown in Figure 1 for the initial values $x_{1}(0)=2.3$ and $x_{2}(0)=1$

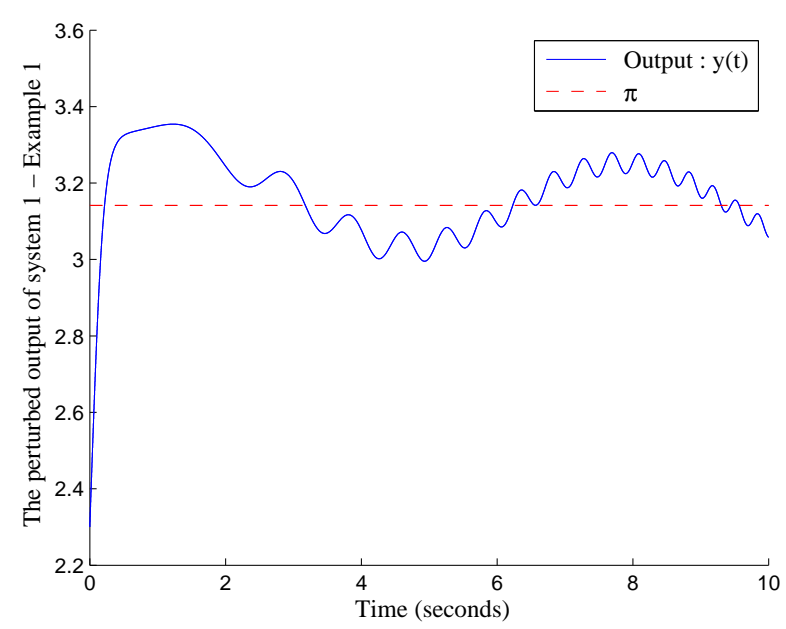

Figure 1: The perturbed output $y(t)$ of system 41]

First, we implement the dynamic extensions $\hat{x}$ and $x_{*}$ given respectively by equations $(12)$ and $(13)$. Then, for different values of the delay $\tau$, we implement the exact estimation of the stat $x$ given by (14).

\section{Numerical examples and simulation results:}

- Choosing $\tau=0.1$, for $t \geq 0.1$, the exact estimation for given by 14 is equivalent to

$$
\begin{aligned}
x(t)= & 10^{4}\left[\begin{array}{ll}
-0.1774 & 0.0995 \\
-3.8992 & 2.1813
\end{array}\right] x_{*}(t)-10^{4}\left[\begin{array}{ll}
-0.1775 & 0.0995 \\
-3.8992 & 2.1812
\end{array}\right] \hat{x}(t) \\
& +10^{4}\left[\begin{array}{ll}
-0.1503 & 0.0831 \\
-3.3007 & 1.8220
\end{array}\right]\left(\hat{x}(t-0.1)-x_{*}(t-0.1)\right) .
\end{aligned}
$$

- Choosing $\tau=0.5$, then

$$
x(t)=\left[\begin{array}{cc}
-66.11 & 38.80 \\
-405.89 & 234.66
\end{array}\right] x_{*}(t)-\left[\begin{array}{cc}
-67.11 & 38.80 \\
-405.89 & 233.66
\end{array}\right] \hat{x}(t)+\left[\begin{array}{cc}
-29.31 & 15.94 \\
-176.86 & 95.51
\end{array}\right]\left(\hat{x}(t-0.5)-x_{*}(t-0.5)\right) .
$$

- Choosing $\tau=1$, then

$$
x(t)=\left[\begin{array}{ll}
-14.61 & 9.31 \\
-64.65 & 39.45
\end{array}\right] x_{*}(t)-\left[\begin{array}{cc}
-15.61 & 9.31 \\
-64.65 & 38.45
\end{array}\right] \hat{x}(t)+\left[\begin{array}{cc}
-3.01 & 1.60 \\
-12.40 & 6.56
\end{array}\right]\left(\hat{x}(t-1)-x_{*}(t-1)\right) .
$$

Figure 2 illustrates these 3 numerical examples. We observe that the estimation is exact for all $t \geq \tau$. 

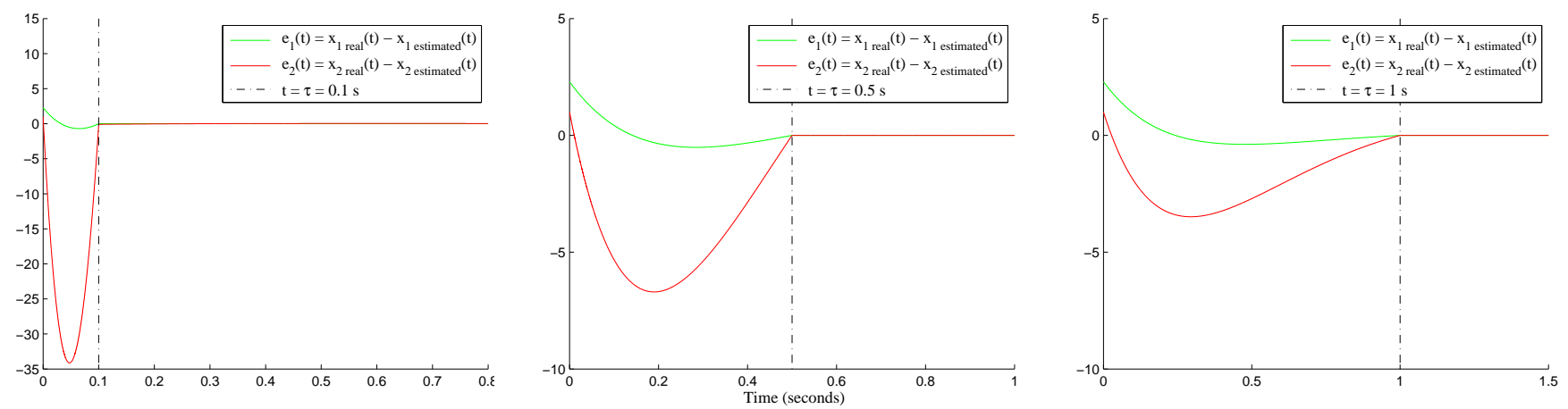

Figure 2: The errors between the real state and the exact estimation for $\tau=0.1, \tau=0.5$ and $\tau=1$

Now, we assume that Assumption B is satisfied and apply Theorem 4. We have

$$
-R_{1} f\left(y, u, \epsilon_{2}\right)=\left[\begin{array}{rc}
-3 & 2 \\
2 & -2
\end{array}\right]\left(\begin{array}{c}
y^{2} \sin (y)+\frac{5}{2} y+\epsilon_{2} \\
y^{2} \sin (y)+\frac{3}{2} y+\epsilon_{2}
\end{array}\right)=\left(\begin{array}{c}
-y^{2} \sin (y)-\frac{9}{2} y-\epsilon_{2} \\
2 y
\end{array}\right) .
$$

Therefore $-R_{1} f\left(y, u, \epsilon_{2}\right)=\varphi_{1}\left(y, \epsilon_{2}, y, \epsilon_{2}\right)$ with

$$
\varphi_{1}\left(a_{1}, a_{2}, b_{1}, b_{2}\right)=\left(\begin{array}{c}
2 a_{1}^{3}+2 a_{1}-a_{1}^{2} \sin \left(a_{1}\right)-2 b_{1}^{3}-\frac{13}{2} b_{1}-b_{2} \\
2 a_{1}
\end{array}\right) .
$$

We also have

$$
R_{2} f\left(y, u, \epsilon_{2}\right)=\left[\begin{array}{rc}
-2 & 1 \\
3 & -1
\end{array}\right]\left(\begin{array}{c}
y^{2} \sin (y)+\frac{5}{2} y+\epsilon_{2} \\
y^{2} \sin (y)+\frac{3}{2} y+\epsilon_{2}
\end{array}\right)=\left(\begin{array}{c}
-y^{2} \sin (y)-\frac{7}{2} y-\epsilon_{2} \\
2 y^{2} \sin (y)+6 y+2 \epsilon_{2}
\end{array}\right) .
$$

Therefore $R_{2} f\left(y, u, \epsilon_{2}\right)=\varphi_{2}\left(y, \epsilon_{2}, y, \epsilon_{2}\right)$ with

$$
\varphi_{2}\left(a_{1}, a_{2}, b_{1}, b_{2}\right)=\left(\begin{array}{c}
2 a_{1}^{3}+2 a_{1}-a_{1}^{2} \sin \left(a_{1}\right)-2 b_{1}^{3}-\frac{11}{2} b_{1}-b_{2} \\
2 a_{1}^{3}+8 a_{1}-2 b_{1}^{3}-2 b_{1}+2 a_{1}^{2} \sin \left(a_{1}\right)+2 a_{2}
\end{array}\right) .
$$

Both $\varphi_{1}$ and $\varphi_{2}$ satisfy the required monotonicity properties.

For $i=1,2$, we assume that $\bar{\epsilon}_{i}=-\underline{\epsilon}_{i}$. Then

$$
\begin{aligned}
& \bar{\psi}_{1}(y)=\left(\begin{array}{c}
2\left(y+\bar{\epsilon}_{1}\right)^{3}+2\left(y+\bar{\epsilon}_{1}\right)-\left(y+\bar{\epsilon}_{1}\right)^{2} \sin \left(y+\bar{\epsilon}_{1}\right)-2\left(y-\bar{\epsilon}_{1}\right)^{3}-\frac{13}{2}\left(y-\bar{\epsilon}_{1}\right)-\underline{\epsilon}_{2} \\
2\left(y+\bar{\epsilon}_{1}\right)
\end{array}\right), \\
& \underline{\psi}_{1}(y)=\left(\begin{array}{c}
2\left(y-\bar{\epsilon}_{1}\right)^{3}+2\left(y-\bar{\epsilon}_{1}\right)-\left(y-\bar{\epsilon}_{1}\right)^{2} \sin \left(y-\bar{\epsilon}_{1}\right)-2\left(y+\bar{\epsilon}_{1}\right)^{3}-\frac{13}{2}\left(y+\bar{\epsilon}_{1}\right)-\bar{\epsilon}_{2} \\
2\left(y-\bar{\epsilon}_{1}\right)
\end{array}\right), \\
& \bar{\psi}_{2}(y)=\left(\begin{array}{c}
2\left(y+\bar{\epsilon}_{1}\right)^{3}+2\left(y+\bar{\epsilon}_{1}\right)-\left(y+\bar{\epsilon}_{1}\right)^{2} \sin \left(y+\bar{\epsilon}_{1}\right)-2\left(y-\bar{\epsilon}_{1}\right)^{3}-\frac{11}{2}\left(y-\bar{\epsilon}_{1}\right)-\underline{\epsilon}_{2} \\
2\left(y+\bar{\epsilon}_{1}\right)^{3}+8\left(y+\bar{\epsilon}_{1}\right)-2\left(y-\bar{\epsilon}_{1}\right)^{3}-2\left(y-\bar{\epsilon}_{1}\right)+2\left(y+\bar{\epsilon}_{1}\right)^{2} \sin \left(y+\bar{\epsilon}_{1}\right)+2 \bar{\epsilon}_{2}
\end{array}\right), \\
& \underline{\psi}_{2}(y)=\left(\begin{array}{c}
2\left(y-\bar{\epsilon}_{1}\right)^{3}+2\left(y-\bar{\epsilon}_{1}\right)-\left(y-\bar{\epsilon}_{1}\right)^{2} \sin \left(y-\bar{\epsilon}_{1}\right)-2\left(y+\bar{\epsilon}_{1}\right)^{3}-\frac{11}{2}\left(y+\bar{\epsilon}_{1}\right)-\bar{\epsilon}_{2} \\
2\left(y-\bar{\epsilon}_{1}\right)^{3}+8\left(y-\bar{\epsilon}_{1}\right)-2\left(y+\bar{\epsilon}_{1}\right)^{3}-2\left(y+\bar{\epsilon}_{1}\right)+2\left(y-\bar{\epsilon}_{1}\right)^{2} \sin \left(y-\bar{\epsilon}_{1}\right)-2 \bar{\epsilon}_{2}
\end{array}\right) .
\end{aligned}
$$

Now, we have

$$
\begin{gathered}
F_{\tau}=E_{\tau} R_{1}^{-1} e^{-\tau M_{1}}, \\
G_{\tau}=E_{\tau} R_{2}^{-1} e^{-\tau M_{2}}, \\
M_{3}=\left[\begin{array}{rc}
-\frac{1}{2} & 0 \\
0 & -\frac{1}{3}
\end{array}\right]\left[\begin{array}{rc}
e^{-2 \tau}-1 & 0 \\
0 & e^{-3 \tau}-1
\end{array}\right]=\left[\begin{array}{cc}
\frac{1-e^{-2 \tau}}{2} & 0 \\
0 & \frac{1-e^{-3 \tau}}{3}
\end{array}\right] .
\end{gathered}
$$

We implement the dynamic extensions $z_{a}, z_{b}, z_{c}, z_{d}$ and $z_{e}$ given by equations (34), as well as the lower and upper bounds dynamics given by (35). The following figures illustrate our results for several values of $\bar{\epsilon}_{1}, \bar{\epsilon}_{2}$ and $\tau$. 

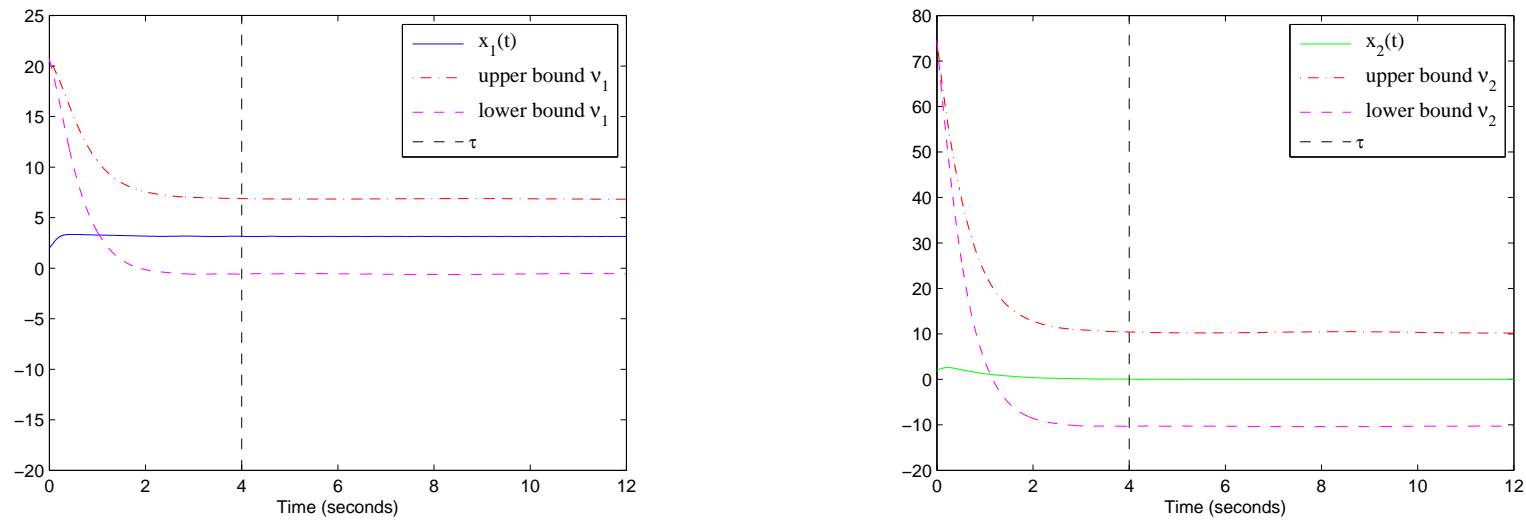

Figure 3: Finite time interval estimation with upper and lower bounds for $\tau=4, \bar{\epsilon}_{1}=0.02, \bar{\epsilon}_{2}=0.2$.
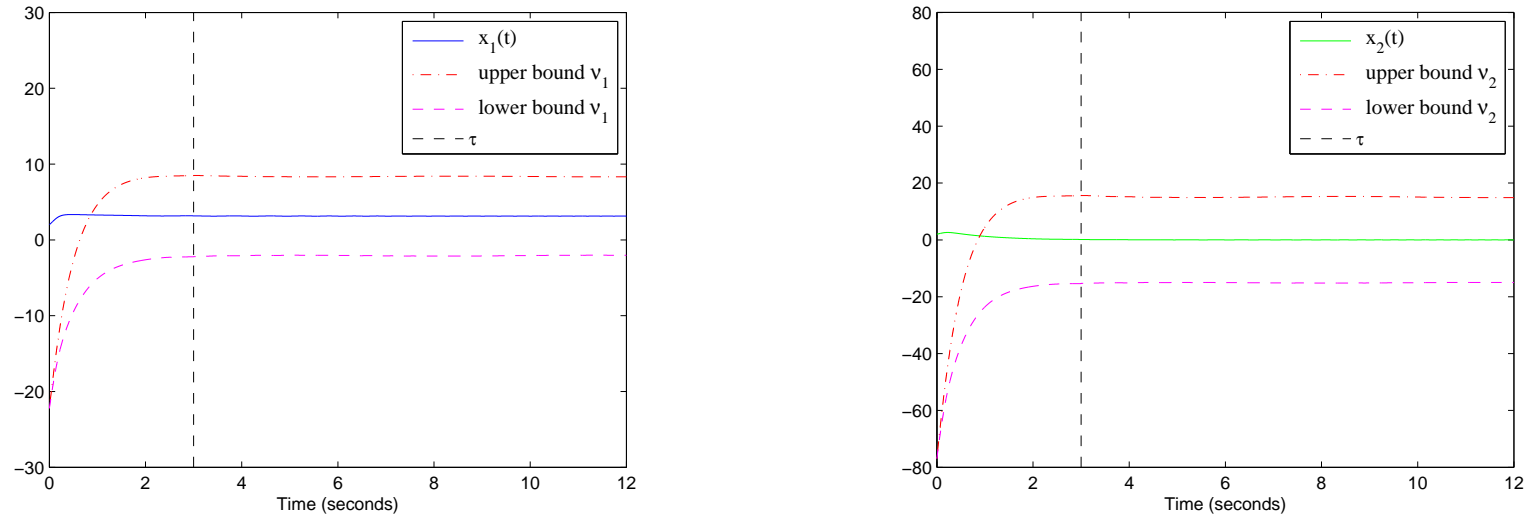

Figure 4: Finite time interval estimation with upper and lower bounds for $\tau=3, \bar{\epsilon}_{1}=0.01, \bar{\epsilon}_{2}=0.02$.

\subsection{Second example}

In this section, we illustrate our results with the model of electromechanical system described in [6]:

$$
\left\{\begin{array}{l}
\dot{x}_{1}=x_{2} \\
\dot{x}_{2}=b_{1} x_{3}-a_{1} \sin \left(x_{1}\right)-a_{2} x_{2} \\
\dot{x}_{3}=b_{0} u-a_{3} x_{2}-a_{4} x_{3}
\end{array}\right.
$$

with $x=\left(x_{1}, x_{2}, x_{3}\right) \in \mathbb{R}^{3}$, with the output $y=x_{1}$ and where the $b_{i}$ 's and the $a_{i}$ 's are positive real numbers. Assuming that the variable $x_{1}$ is measured is realistic from an applied point of view because $x_{1}$ represents the angular motor position of the device.

We let $b_{0}=40, b_{1}=15, a_{1}=35, a_{2}=1, a_{3}=36.4$ and $a_{4}=200$. These values are close to the numerical values given in [6]. With the notation of the previous sections, we can choose

$$
A=\left[\begin{array}{ccc}
-2 & 1 & 0 \\
0 & -1 & 15 \\
0 & -36.4 & -200
\end{array}\right] \quad, \quad f(y, u)=\left(\begin{array}{c}
2 y \\
-35 \sin (y) \\
40 u
\end{array}\right)
$$


Selecting

$$
L=-\left(\begin{array}{l}
8 \\
1 \\
1
\end{array}\right)
$$

we obtain

$$
H=\left[\begin{array}{ccl}
-10 & 1 & 0 \\
-1 & -1 & 15 \\
-1 & -36.4 & -200
\end{array}\right]
$$

Then

$$
M_{1}=R_{1} A R_{1}^{-1}=\left[\begin{array}{ccc}
-2 & 0 & 0 \\
0 & -3.7826 & 0 \\
0 & 0 & -197.2174
\end{array}\right]
$$

and

$$
M_{2}=R_{2} H R_{2}^{-1}=\left[\begin{array}{ccc}
-9.8186 & 0 & 0 \\
0 & -3.9635 & 0 \\
0 & 0 & -197.2179
\end{array}\right]
$$

with

$$
R_{1}=\left[\begin{array}{lll}
1 & 0.5690 & 0.0431 \\
0 & 1.1782 & 0.0901 \\
0 & 0.1887 & 1.0173
\end{array}\right] \quad, \quad R_{2}=\left[\begin{array}{ccc}
-1.0485 & 0.1763 & 0.0139 \\
-0.1922 & 1.0779 & 0.0825 \\
0.0064 & 0.1887 & 1.0173
\end{array}\right]
$$

Choosing $\tau=0.1$, Theorem 2 gives the exact estimate of the state $x(t)$ for $t \geq 0.1$ :

$$
\begin{aligned}
x(t)= & 10^{3}\left[\begin{array}{ccc}
0.0032 & 0.0159 & 0.1891 \\
0.4214 & -2.5809 & 6.0261 \\
-0.0782 & 0.4788 & -1.1179
\end{array}\right] x_{*}(t)-10^{3}\left[\begin{array}{ccc}
0.0022 & 0.0159 & 0.1891 \\
0.4214 & -2.5819 & 6.0261 \\
-0.0782 & 0.4788 & -1.1189
\end{array}\right] \hat{x}(t) \\
& +10^{3}\left[\begin{array}{ccc}
0.0018 & -0.0131 & -0.0010 \\
0.3450 & -2.5390 & -0.1942 \\
-0.0640 & 0.4710 & 0.0360
\end{array}\right]\left(\hat{x}(t-0.1)-x_{*}(t-0.1)\right)
\end{aligned}
$$

where $\hat{x}(t)$ and $x_{*}(t)$ are the solutions of the dynamic extensions given by 12 and 13 with the matrices and functions we have selected in this section. We considered the case where the input is $u(t)=(2 t+1) \sin (50 t)$ and obtained the simulations:
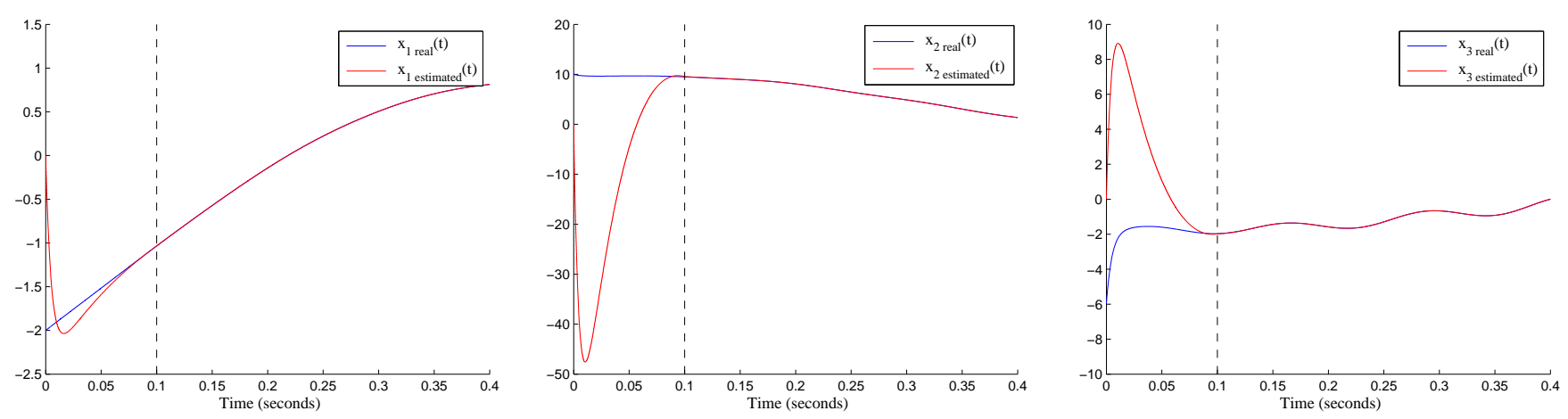

Figure 5: The real states and the exact estimations for $\tau=0.1 s$. 
Now, we add to the example 64 some disturbances: we consider:

$$
\left\{\begin{aligned}
\dot{x}_{1} & =x_{2}+\epsilon_{2} \\
\dot{x}_{2} & =b_{1} x_{3}-a_{1} \sin \left(x_{1}\right)-a_{2} x_{2}+\epsilon_{2} \\
\dot{x}_{3} & =b_{0} u-a_{3} x_{2}-a_{4} x_{3}+\epsilon_{2} \\
y & =x_{1}+\epsilon_{1}
\end{aligned}\right.
$$

under Assumption B. Moreover, for the numerical implementation, we suppose that, for all $t \geq 0$ and $i \in\{1,2\}, \bar{\epsilon}_{i} \geq 0$, $\epsilon_{i}(t)=\bar{\epsilon}_{i} \sin \left(t^{2}\right)$ and $\underline{\epsilon}_{i}=-\bar{\epsilon}_{i}$.

For the values of $a_{1}, a_{2}, a_{3}, a_{4}, b_{0}, b_{1}$ indicated above and the matrix $A$ given in (65), the corresponding function $f$ is

$$
f\left(y, u, \epsilon_{2}\right)=\left(\begin{array}{c}
2 y+\epsilon_{2} \\
-35 \sin (y)+\epsilon_{2} \\
40 u+\epsilon_{2}
\end{array}\right) .
$$

Then

$$
-R_{1} f\left(y, u, \epsilon_{2}\right)=\left[\begin{array}{c}
-2 y+19.9138 \sin (y)-1.7241 u-1.6121 \epsilon_{2} \\
41.2376 \sin (y)-3.6028 u-1.2683 \epsilon_{2} \\
6.6054 \sin (y)-40.6938 u-1.2061 \epsilon_{2}
\end{array}\right]
$$

and

$$
R_{2} f\left(y, u, \epsilon_{2}\right)=\left[\begin{array}{c}
-2.0971 y-6.1702 \sin (y)+0.5562 u-0.8583 \epsilon_{2} \\
-0.3844 y-37.7256 \sin (y)+3.2990 u+0.9681 \epsilon_{2} \\
0.0129 y-6.6042 \sin (y)+40.6936 u+1.2125 \epsilon_{2}
\end{array}\right] .
$$

We choose

$$
\varphi_{1}\left(a_{1}, a_{2}, b_{1}, b_{2}, u\right)=\left[\begin{array}{c}
20 a_{1}+19.9138 \sin \left(a_{1}\right)-22 b_{1}-1.7241 u-1.6121 b_{2} \\
41.5 a_{1}+41.2376 \sin \left(a_{1}\right)-41.5 b_{1}-3.6028 u-1.2683 b_{2} \\
7 a_{1}+6.6054 \sin \left(a_{1}\right)-7 b_{1}-40.6938 u-1.2061 b_{2}
\end{array}\right],
$$

and

$$
\varphi_{2}\left(a_{1}, a_{2}, b_{1}, b_{2}, u\right)=\left[\begin{array}{c}
6.5 a_{1}-6.1702 \sin \left(a_{1}\right)-8.5971 b_{1}+0.5562 u-0.8583 b_{2} \\
38 a_{1}-37.7256 \sin \left(a_{1}\right)-38.3844 b_{1}+3.2990 u+0.9681 a_{2} \\
6.8129 a_{1}-6.6042 \sin \left(a_{1}\right)-6.8 b_{1}+40.6936 u+1.2125 a_{2}
\end{array}\right],
$$

Notice that $\varphi_{1}$ and $\varphi_{2}$ satisfy the required monotonicity properties. As an immediate consequence of our definitions, we have

$$
\begin{gathered}
\bar{\psi}_{1}(y, u)=\left[\begin{array}{c}
20\left(y+\bar{\epsilon}_{1}\right)+19.9138 \sin \left(y+\bar{\epsilon}_{1}\right)-22\left(y-\bar{\epsilon}_{1}\right)-1.7241 u-1.6121 \underline{\epsilon}_{2} \\
41.5\left(y+\bar{\epsilon}_{1}\right)+41.2376 \sin \left(y+\bar{\epsilon}_{1}\right)-41.5\left(y-\bar{\epsilon}_{1}\right)-3.6028 u-1.2683 \underline{\epsilon}_{2} \\
7\left(y+\bar{\epsilon}_{1}\right)+6.6054 \sin \left(\left(y+\bar{\epsilon}_{1}\right)\right)-7\left(y-\bar{\epsilon}_{1}\right)-40.6938 u-1.2061 \underline{\epsilon}_{2}
\end{array}\right], \\
\underline{\psi}_{1}(y, u)=\left[\begin{array}{c}
20\left(y-\bar{\epsilon}_{1}\right)+19.9138 \sin \left(y-\bar{\epsilon}_{1}\right)-22\left(y+\bar{\epsilon}_{1}\right)-1.7241 u-1.6121 \bar{\epsilon}_{2} \\
41.5\left(y-\bar{\epsilon}_{1}\right)+41.2376 \sin \left(y-\bar{\epsilon}_{1}\right)-41.5\left(y+\bar{\epsilon}_{1}\right)-3.6028 u-1.2683 \bar{\epsilon}_{2} \\
7\left(y-\bar{\epsilon}_{1}\right)+6.6054 \sin \left(\left(y-\bar{\epsilon}_{1}\right)\right)-7\left(y+\bar{\epsilon}_{1}\right)-40.6938 u-1.2061 \bar{\epsilon}_{2}
\end{array}\right], \\
\bar{\psi}_{2}(y, u)=\left[\begin{array}{c}
6.5\left(y+\bar{\epsilon}_{1}\right)-6.1702 \sin \left(y+\bar{\epsilon}_{1}\right)-8.5971\left(y-\bar{\epsilon}_{1}\right)+0.5562 u-0.8583 \underline{\epsilon}_{2} \\
38\left(y+\bar{\epsilon}_{1}\right)-37.7256 \sin \left(y+\bar{\epsilon}_{1}\right)-38.3844\left(y-\bar{\epsilon}_{1}\right)+3.2990 u+0.9681 \bar{\epsilon}_{2} \\
6.8129\left(y+\bar{\epsilon}_{1}\right)-6.6042 \sin \left(y+\bar{\epsilon}_{1}\right)-6.8\left(y-\bar{\epsilon}_{1}\right)+40.6936 u+1.2125 \bar{\epsilon}_{2}
\end{array}\right], \\
\underline{\psi}_{2}(y, u)=\left[\begin{array}{c}
6.5\left(y-\bar{\epsilon}_{1}\right)-6.1702 \sin \left(y-\bar{\epsilon}_{1}\right)-8.5971\left(y+\bar{\epsilon}_{1}\right)+0.5562 u-0.8583 \bar{\epsilon}_{2} \\
38\left(y-\bar{\epsilon}_{1}\right)-37.7256 \sin \left(y-\bar{\epsilon}_{1}\right)-38.3844\left(y+\bar{\epsilon}_{1}\right)+3.2990 u+0.9681 \underline{\epsilon}_{2} \\
6.8129\left(y-\bar{\epsilon}_{1}\right)-6.6042 \sin \left(y-\bar{\epsilon}_{1}\right)-6.8\left(y+\bar{\epsilon}_{1}\right)+40.6936 u+1.2125 \underline{\epsilon}_{2}
\end{array}\right] .
\end{gathered}
$$

Finally we implement the dynamic extensions $z_{a}, z_{b}, z_{c}, z_{d}$ and $z_{e}$ given by equations (34), and the lower and upper bounds dynamics given by (35). The following figures illustrate an example where $\tau=0.1, \bar{\epsilon}_{1}=0.001, \bar{\epsilon}_{2}=0.02$ and the input $u(t)=(2 t+1) \sin (50 t)$. 

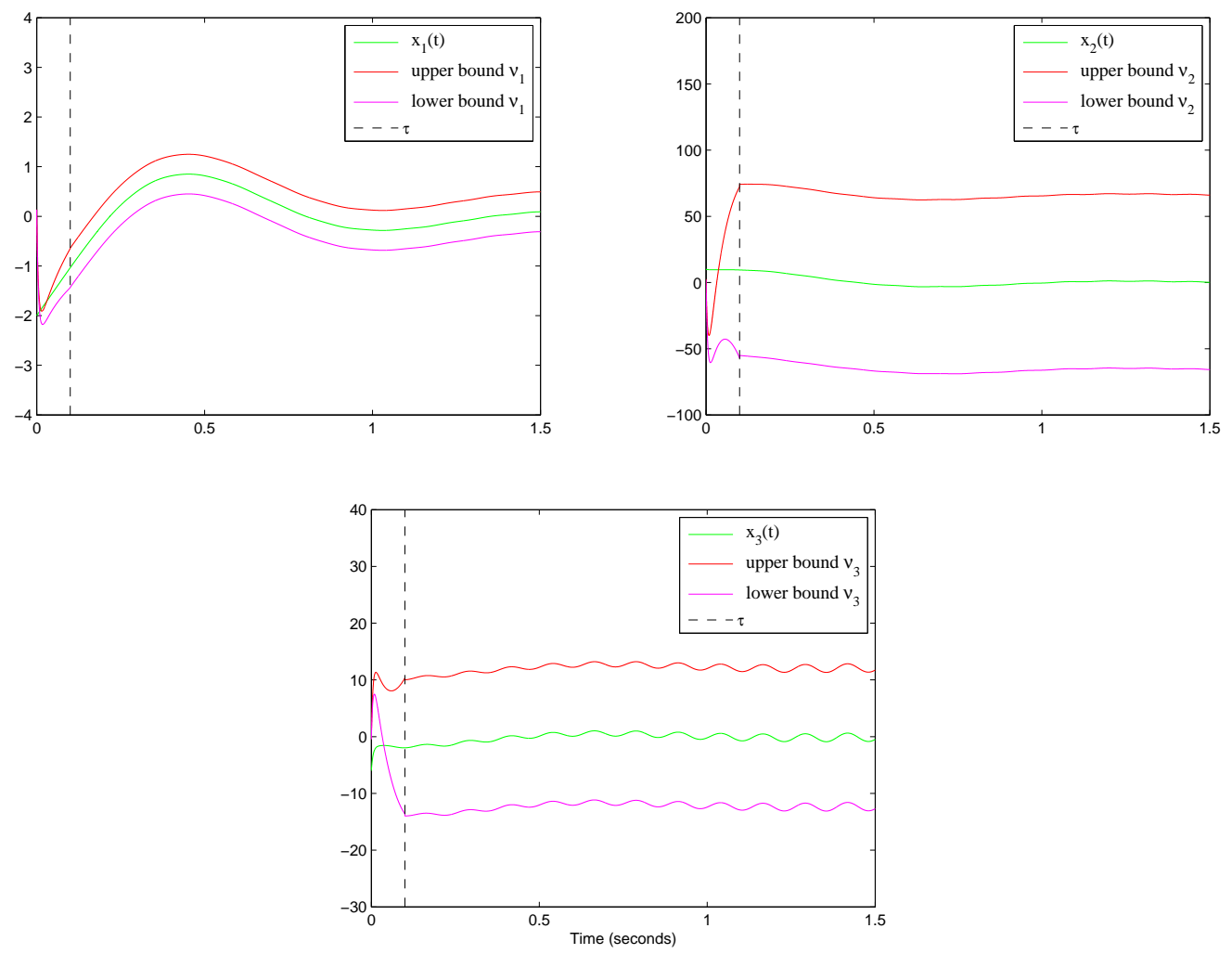

Figure 6: Example 2 with $\tau=0.1 s, u(t)=(2 t+1) \sin (50 t), \bar{\epsilon}_{1}=0.001$ and $\bar{\epsilon}_{2}=0.02$

\subsection{Third example}

In order to show that the size of the estimation intervals provided by the observer (34), i.e. its performance, strongly depends on the size of the entries of the matrix $A$ and on the type of nonlinearities present in the dynamics of the studied system, we consider:

$$
\left\{\begin{aligned}
\dot{x}_{1} & =-x_{1}+x_{2}+\epsilon_{2} \\
\dot{x}_{2} & =-\frac{x_{1}}{2} \cos \left(x_{1}\right)+u+\epsilon_{2} \\
y & =x_{1}+\epsilon_{1}
\end{aligned}\right.
$$

Now, let us apply Theorem 4. We can choose

$$
A=\left[\begin{array}{ll}
-\frac{5}{2} & 1 \\
-1 & 0
\end{array}\right], \quad f\left(y, u, \epsilon_{2}\right)=\left(\begin{array}{c}
\frac{3}{2} y+\epsilon_{2} \\
y-\frac{y}{2} \cos (y)+u+\epsilon_{2}
\end{array}\right)
$$

We observe that the nonlinear term $\frac{y}{2} \cos (y)$ is not globally Lipschitz, but bounded in norm by a linear function of the norm. We observe also that the entries of $A$ are neither very small nor very big. Since $C=\left[\begin{array}{ll}1 & 0\end{array}\right]$, the pair $(A, C)$ is observable. Moreover, the matrix $A$ is Hurwitz and the function $f$ is of class $C^{1}$. For $L=-\left(\begin{array}{c}1 \\ 0.4\end{array}\right)$, we obtain

$$
H=\left[\begin{array}{ll}
-3.5 & 1 \\
-1.4 & 0
\end{array}\right]
$$

One can check readily that:

$$
R_{1} A R_{1}^{-1}=\left[\begin{array}{rc}
-2 & 0 \\
0 & -\frac{1}{2}
\end{array}\right]=M_{1} \quad \text { and } \quad R_{2} H R_{2}^{-1}=\left[\begin{array}{rc}
-3.039 & 0 \\
0 & -0.460
\end{array}\right]=M_{2}
$$


with

$$
R_{1}=\frac{\sqrt{5}}{3}\left[\begin{array}{rc}
-2 & 1 \\
1 & -2
\end{array}\right] \quad \text { and } \quad R_{2}=\left[\begin{array}{rc}
-1.297 & 0.426 \\
0.571 & -1.240
\end{array}\right]
$$

Consequently,

$$
-R_{1} f\left(y, 0, \epsilon_{2}\right)=\frac{\sqrt{5}}{3}\left(\begin{array}{c}
2 y+\frac{y}{2} \cos (y)+\epsilon_{2} \\
\frac{y}{2}-y \cos (y)+\epsilon_{2}
\end{array}\right) .
$$

Thus $-R_{1} f\left(y, 0, \epsilon_{2}\right)=\varphi_{1}\left(y, \epsilon_{2}, y, \epsilon_{2}\right)$ with

$$
\varphi_{1}\left(a_{1}, a_{2}, b_{1}, b_{2}\right)=\frac{\sqrt{5}}{3}\left(\begin{array}{c}
\frac{a_{1}^{3}}{6}+2 a_{1}+\frac{a_{1}}{2} \cos \left(a_{1}\right)-\frac{b_{1}^{3}}{6}+a_{2} \\
a_{1}^{3}+2 a_{1}-a_{1} \cos \left(a_{1}\right)-b_{1}^{3}-\frac{3}{2} b_{1}+a_{2}
\end{array}\right) .
$$

We also have

$$
R_{2} f\left(y, 0, \epsilon_{2}\right)=\left(\begin{array}{l}
-1.5195 y-0.2135 y \cos (y)-0.8707 \epsilon_{2} \\
-0.3835 y+0.6204 y \cos (y)-0.6692 \epsilon_{2}
\end{array}\right)
$$

Therefore $R_{2} f\left(y, 0, \epsilon_{2}\right)=\varphi_{2}\left(y, \epsilon_{2}, y, \epsilon_{2}\right)$ with

$$
\varphi_{2}\left(a_{1}, a_{2}, b_{1}, b_{2}\right)=\left(\begin{array}{c}
\frac{a_{1}^{3}}{12}-\frac{b_{1}^{3}}{12}-1.5195 b_{1}-0.2135 b_{1} \cos \left(b_{1}\right)-0.8707 b_{2} \\
a_{1}^{3} \\
\frac{3}{3}+a_{1}-\frac{b_{1}^{3}}{3}-1.3835 b_{1}+0.6204 b_{1} \cos \left(b_{1}\right)-0.6692 b_{2}
\end{array}\right) .
$$

Both $\varphi_{1}$ and $\varphi_{2}$ satisfy the required monotonicity properties. Moreover we assume that $\bar{\epsilon}_{i}=-\underline{\epsilon}_{i}$, for $i=1,2$. Then we implement the dynamic extensions $z_{a}, z_{b}, z_{c}, z_{d}$ and $z_{e}$, as well as the lower and upper bounds. The following figures illustrate our results for $\bar{\epsilon}_{1}=0.1, \bar{\epsilon}_{2}=0.2$ and $\tau=4$.
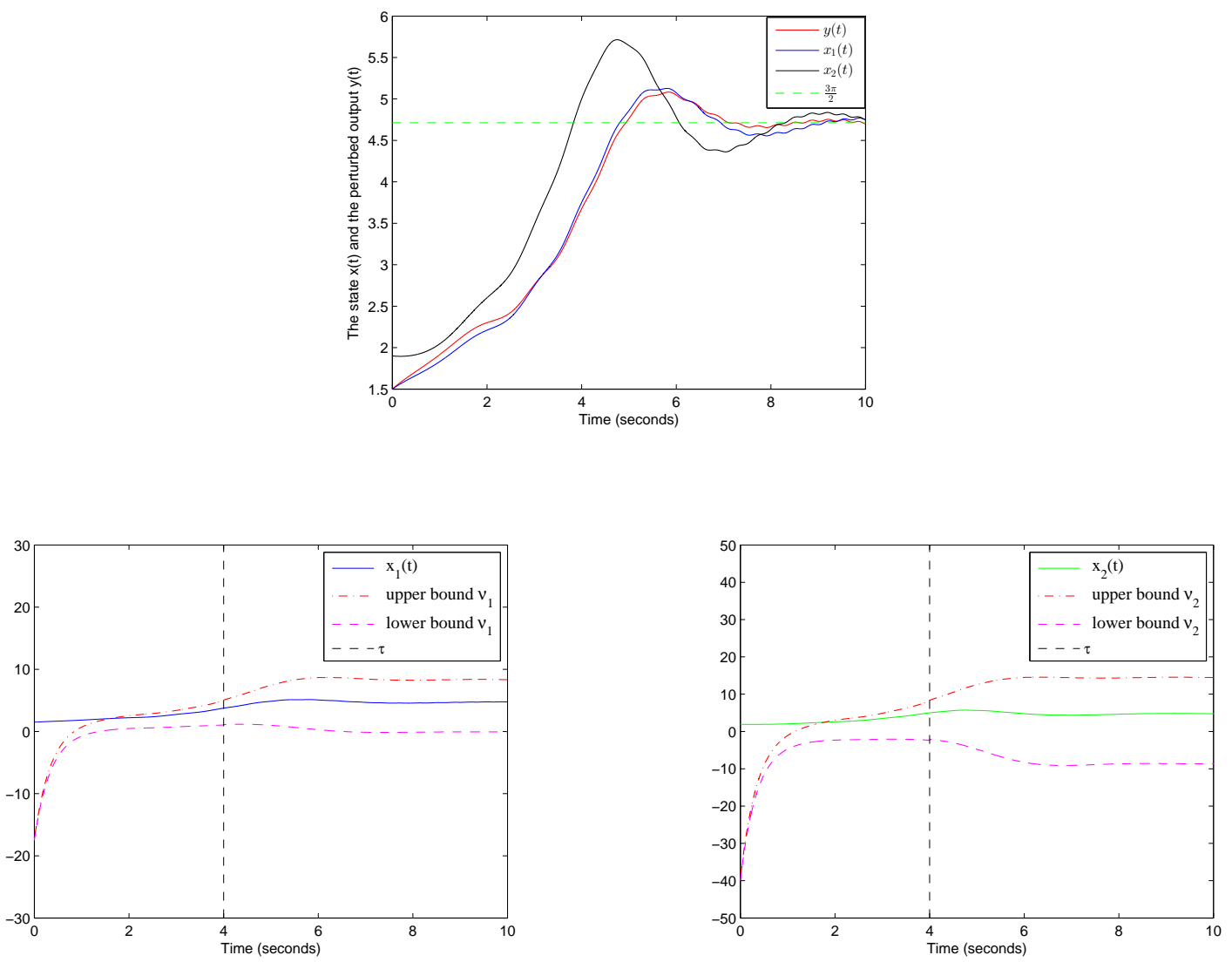

Figure 7: Finite time interval estimation with upper and lower bounds for $\tau=4, \bar{\epsilon}_{1}=0.1, \bar{\epsilon}_{2}=0.2$. 
In this example, although the disturbances on the output are considerably stronger than the ones of Example 1 (five times greater, see Figure 3), it turns out that the performance, in terms of size of estimation interval, of the observer is better than the one of the observer we proposed for the Example 1. We observe also that Example 2 leads to matrices $A$ and $H$ with rather large entries, which produce matrices $F_{\tau}, G_{\tau}$ and $E_{\tau} e^{-\tau H}$ with large entries, (between 0 and 62055). This fact is the reason why the performance of the observer for Example 2 is worst than the one of the observer we propose for Example 3. We conclude that the performance of the observers we propose strongly depends on the nature of the observed system, but certainly also on the selections of $\tau, A, H$ and on the choices of functions $\varphi_{1}$ and $\varphi_{2}$.

\section{Conclusion}

We have developed a new technique of estimation of the solutions of nonlinear continuous-time observable systems. Its key features are that it applies in the absence of the knowledge of bounds on the initial conditions and provides, after a finite time interval, with the exact value of the solutions if there is no disturbances and with a lower and an upper bounds when disturbances are present. We conjecture that these results can be used to solve output feedback stabilization of systems. We plan to extend our results to discrete-time systems.

\section{References}

[1] D. P. Bertsekas, I. B. Rhodes, Recursive state estimation for a setmembership description of uncertainty. 16(2) : pp. 117-128, 1971.

[2] F. L. Chernousko, Optimal guaranteed estimates of indeterminacies with the aid of ellipsoids. Engrg. Cybernetics, 18: pp. 1-9, 1980.

[3] C. Combastel, S. A. Raka, A Stable Interval Observer for LTI Systems with No Multiple Poles. 18th IFAC World Congress, Milano, Italy, Aug. 28-Sept. 2, 2011.

[4] R. Engel, G. Kreisselmeier, A Continuous-Time Observer Which Converges in Finite Time. IEEE Trans. on Aut. Contr., Vol. 47, No. 7, July, pp. 1202-1204, 2002.

[5] G. Besançon, An Overview on Observer Tools for Nonlinear Systems. Nonlinear Observers and Applications, Lecture Notes in Control and Information Sciences Volume 363, 2007, pp. 1-33, Springer-Verlag.

[6] D. M. Dawson, J. J. Carroll, M. Schneider, Integrator Backstepping Control of a Brush DC Motor Turning a Robotic Load. IEEE Trans. on Aut. Contr. Systems Technology, vol. 2, No. 3, Sept. 1994, pp. 233-244.

[7] T. N. Dinh, F. Mazenc, S. I. Niculescu, Interval Observer Composed of Observers for Nonlinear Systems, European Control Conference 2014, Strasbourg, France, pp. 660-665.

[8] J.-L. Gouzé, A. Rapaport, Z. Hadj-Sadok, Interval observers for uncertain biological systems. Ecological Modelling, 133, pp. 45-56, 2000.

[9] W. M. Haddad, V. Chellaboina, Q. Hui, Nonnegative and Compartmental Dynamical Systems. Princeton Univ. Press, 2010.

[10] A. A. Kurzhanski, I. Valyi, Ellipsoidal Calculus for Estimation and Control. Birkhauser, Boston, MA, 1997.

[11] Y. Li, R. Sanfelice, A Robust Finite-time Convergent Hybrid Observer for Linear Systems. IEEE 52nd Annual Conference on Decision and Control, pp. 3349-3354, Florence, Italy, Dec. 2013.

[12] J. Liu, S. Laghrouche, M. Wack, Finite time observer design for a class of nonlinear systems with unknown inputs. 2013 American Control Conference, Washington, DC, USA, June 17-19, pp. 286-291, 2013.

[13] D. Luenberger, Observers for multivariable systems. IEEE Trans. Aut. Contr., Vol. 11 (2), pp. 190-197, 1966. 
[14] F. Mazenc, V. Andrieu, M. Malisoff, Design of Continuous-Discrete Observers for Time-Varying Nonlinear Systems. Submitted.

[15] F. Mazenc, O. Bernard, Interval observers for linear time-invariant systems with disturbances, Automatica, Vol. 47, pp. 140-147, 2011.

[16] F. Mazenc, S. I. Niculescu, O. Bernard, Exponentially Stable Interval Observers for Linear Systems with Delay. SIAM J. Control Optim., Vol. 50, pp. 286-305, 2012.

[17] F. Mazenc, T. N. Dinh, S.I. Niculescu, Interval Observers for Discrete-time Systems, International Journal of Robust and Nonlinear Control, Vol. 24, Issue 17, pp. 2867-2890, 2014.

[18] M. Milanese, A. Vicino, Optimal estimation theory for dynamic systems with set membership uncertainty: An overview. Automatica, 27 (6), pp. 997-1009, 1991.

[19] W. Perruquetti, T. Floquet, E. Moulay, Finite-time observers: application to secure communication, IEEE Trans. on Aut. Contr., Vol. 53, No. 1, pp. 356-360, 2008.

[20] T. Raff, F. Allgower, An Impulsive Observer that Estimates the Exact State of a Linear Continuous-Time System in Predetermined Finite Time. Proceedings of the 15th Mediterranean Conference on Control and Automation, July 27-29, Athens, Greece 2007.

[21] T. Raissi, D. Efimov, A. Zolghadri, Interval State Estimation for a Class of Nonlinear Systems. IEEE Trans. on Aut. Contr., Vol. 57, Issue 1, pp. 260-265, Jan. 2012.

[22] F. Sauvage, M. Guay, D. Dochain, Design of a nonlinear finite-time converging observer for a class of nonlinear systems, Journal of Control Science and Engineering, 2007.

[23] F. C. Schweppe, Recursive state estimation: unknown but bounded errors and system inputs. IEEE Trans. on Aut. Contr., Vol. 13, pp. 22-28, Feb, 1968.

[24] R. E. H. Thabet, T. Raissi, C. Combastel, D. Efimov, A. Zolghadri, An effective method to interval observer design for time-varying systems. Automatica, 50 (10), pp. 2677-2684, 2014.

\section{A Expressions of $\epsilon_{L}$ and $\epsilon_{S}$ in Inequalities (33)}

From (3), we deduce that the function $\epsilon_{3}$ defined in $(23)$ satisfies, for all $t \geq 0$,

$$
\underline{\epsilon}_{3} \leq \epsilon_{3}(t) \leq \bar{\epsilon}_{3}
$$

with

$$
\begin{aligned}
& \bar{\epsilon}_{3}=\left(e^{-\tau M_{2}} R_{2} L\right)^{+} \bar{\epsilon}_{1}-\left(e^{-\tau M_{2}} R_{2} L\right)^{-} \underline{\epsilon}_{1}, \\
& \underline{\epsilon}_{3}=\left(e^{-\tau M_{2}} R_{2} L\right)^{+} \underline{\epsilon}_{1}-\left(e^{-\tau M_{2}} R_{2} L\right)^{-} \bar{\epsilon}_{1} .
\end{aligned}
$$

Since $M_{2}$ is Metzler, the inequalities

$$
\int_{0}^{\tau} e^{l M_{2}} d l \underline{\epsilon}_{3} \leq \int_{t-\tau}^{t} e^{(t-l) M_{2}} \epsilon_{3}(l) d l \leq \int_{0}^{\tau} e^{l M_{2}} d l \bar{\epsilon}_{3}
$$

are satisfied. We deduce that

$$
\begin{aligned}
& \left(E_{\tau} R_{2}^{-1}\right)^{+} M_{3} \underline{\epsilon}_{3} \leq\left(E_{\tau} R_{2}^{-1}\right)^{+} \int_{t-\tau}^{t} e^{(t-l) M_{2}} \epsilon_{3}(l) d l \leq\left(E_{\tau} R_{2}^{-1}\right)^{+} M_{3} \bar{\epsilon}_{3}, \\
& \left(E_{\tau} R_{2}^{-1}\right)^{-} M_{3} \underline{\epsilon}_{3} \leq\left(E_{\tau} R_{2}^{-1}\right)^{-} \int_{t-\tau}^{t} e^{(t-l) M_{2}} \epsilon_{3}(l) d l \leq\left(E_{\tau} R_{2}^{-1}\right)^{-} M_{3} \bar{\epsilon}_{3},
\end{aligned}
$$

with $M_{3}=M_{2}^{-1}\left(e^{\tau M_{2}}-I\right)$. As an immediate consequence,

$$
\left(E_{\tau} R_{2}^{-1}\right)^{+} M_{3} \underline{\epsilon}_{3}-\left(E_{\tau} R_{2}^{-1}\right)^{-} M_{3} \bar{\epsilon}_{3} \leq E_{\tau} R_{2}^{-1} \int_{t-\tau}^{t} e^{(t-l) M_{2}} \epsilon_{3}(l) d l \leq\left(E_{\tau} R_{2}^{-1}\right)^{+} M_{3} \bar{\epsilon}_{3}-\left(E_{\tau} R_{2}^{-1}\right)^{-} M_{3} \underline{\epsilon}_{3} .
$$

It follows that

$$
\epsilon_{S} \leq E_{\tau} R_{2}^{-1} \int_{t-\tau}^{t} e^{(t-l) M_{2}} \epsilon_{3}(l) d l \leq \epsilon_{L}
$$


with

$$
\begin{aligned}
\epsilon_{L} & =\left(E_{\tau} R_{2}^{-1}\right)^{+} M_{3} \bar{\epsilon}_{3}-\left(E_{\tau} R_{2}^{-1}\right)^{-} M_{3} \underline{\epsilon}_{3}, \\
\epsilon_{S} & =\left(E_{\tau} R_{2}^{-1}\right)^{+} M_{3} \underline{\epsilon}_{3}-\left(E_{\tau} R_{2}^{-1}\right)^{-} M_{3} \bar{\epsilon}_{3} .
\end{aligned}
$$

Using the expressions of $\bar{\epsilon}_{3}$ and $\underline{\epsilon}_{3}$, we obtain 27 .

Remark 2. In the particular case where $\bar{\epsilon}_{1}=-\underline{\epsilon}_{1}$, the expressions of $\epsilon_{L}$ and $\epsilon_{S}$ simplify as

$$
\begin{aligned}
\epsilon_{L} & =\left[\left(E_{\tau} R_{2}^{-1}\right)^{+}+\left(E_{\tau} R_{2}^{-1}\right)^{-}\right] M_{3}\left[\left(e^{-\tau M_{2}} R_{2} L\right)^{+}+\left(e^{-\tau M_{2}} R_{2} L\right)^{-}\right] \bar{\epsilon}_{1}, \\
\epsilon_{S} & =-\epsilon_{L}
\end{aligned}
$$

\section{B Technical lemmas}

\section{B.1 First result}

Lemma 1. Let $(A, C)$ be an observable pair. Then there is a matrix $L$ such that the matrix $H=A+L C$ is Hurwitz and there is $\tau_{*}$ such that, for all $\tau \geq \tau_{*}$, the matrix $e^{-\tau H}-e^{-\tau A}$ is invertible.

Proof. Since the pair $(A, C)$ is observable, there is a matrix $L$ so that all the eigenvalues of $H$ are real, negative and smaller than the smallest of the real parts of the eigenvalues of $-A$. Then $\lim _{r \rightarrow+\infty}\left|e^{H r}\right|\left|e^{-A r}\right|=0$. Consequently, there is $\tau_{*}>0$ such that, for all $\tau \geq \tau_{*},\left|e^{H \tau}\right|\left|e^{-A \tau}\right|<1$. Now, observe that the matrix $e^{-\tau H}-e^{-\tau A}$ is invertible if and only if $e^{\tau H} e^{-\tau A}-I$ is invertible. Since $\left|e^{H \tau}\right|\left|e^{-A \tau}\right|<1$, it does not exist a vector $V \neq 0$ such that $\left(e^{\tau H} e^{-\tau A}-I\right) V=0$. Therefore $e^{\tau H} e^{-\tau A}-I$ is invertible. This conclude the proof.

\section{B.2 Second result}

We recall Lemma 6 of [17].

Lemma 2. Let $g: \mathbb{R}^{k} \rightarrow \mathbb{R}$ be a function of class $C^{1}$. Then there exists a function $g_{c}: \mathbb{R}^{k} \times \mathbb{R}^{k} \rightarrow \mathbb{R}$ nondecreasing with respect to each of its $k$ first variables and nonincreasing with respect to each of its $k$ last variables such that, for all $x \in \mathbb{R}^{k}$, the equality

$$
g_{c}(x, x)=g(x)
$$

is satisfied. 